\title{
SELF-INITIATED USE OF THOUGHT SUBSTITUTION CAN LEAD TO LONG TERM FORGETTING
}

\author{
Chie HOTTA $^{1)}$ and Jun KAWAGUCHI ${ }^{2)}$ \\ I) Shoin Higashi Women's Junior College, Japan, ${ }^{2)}$ Nagoya University, Japan
}

\begin{abstract}
Recent studies indicate that intentionally suppressing the retrieval of an unwanted memory can impair its later recall. However, it is not clear how long this intentional suppression effect persists. The aim of the current study was to investigate this question using a Think/No-Think paradigm. After participants studied pairs of unrelated words, the cue words from each pair were presented either 0,4 , or 12 times during the Think/No-Think phase of the experiment. Participants were asked to either avoid thinking of the word associated with the cue (Supression condition) or to recall it (Response condition). Participants were then asked to recall the cued words immediately and after a delay of 24 hours. Recall performance in the 12-cue No-Think condition was worse than in the baseline (0) condition, both immediately and 24 hours later. Moreover, the participants who thought about something other than the target during the No-Think trials showed poorer recall than those who did not do so. These results suggest that the effect of intentional suppression that occurs under conditions of self-initiated thought substitution can last for over 24 hours.
\end{abstract}

Key words: intentional suppression, long-term forgetting, self-initiated thought substitution

Forgetting unwanted experiences can be adaptive in daily life. Therefore, intentional forgetting can be thought of as an important skill that is worth developing. Several empirical studies have indicated that people can put specific memories out of conscious awareness with some degree of attention and effort, a process variously known as forgetting via stopping retrieval (Anderson, 2003; Anderson \& Green, 2001) or directed forgetting (Bjork, 1989).

Anderson and Green (2001) demonstrated that people can put specific memories out of conscious awareness using a "Think/No-Think" paradigm. The procedure in this study consisted of three phases. First, participants studied pairs of weakly-related words. During the Think/No-Think phase, only the cues for the to-be-remembered words were (repeatedly) presented, and participants were asked to either repeatedly avoid thinking of the to-be-remembered response word $(0,1,8$, or 16 times; suppression condition) or to say it aloud (response condition). During a subsequent cued recall phase, participants were asked to recall the corresponding words in response to the cues. Repeatedly avoiding conscious awareness of the response words led to increased forgetting on the recall test. This is called an intentional suppression effect.

Forgetting may occur by stopping the retrieval process, but for how long does the

Correspondence concerning this article should be addressed to Hotta Chie, Shoin Higashi Women's Junior College, 3-1-8 Wakaenishishinmachi Higashi Osaka City 578-0944, Japan (e-mail: c.hotta@shoinhigashi.ac.jp) 
resulting intentional suppression effect persist? This issue is clearly relevant to everyday life because we sometimes may wish to forget unpleasant events or unnecessary information. In such cases, would repeatedly stopping the retrieval process lead to enduring forgetting? Even if an intentional suppression effect can be observed immediately, longer-term effects should be observed if one is to conclude that true forgetting has occurred. It is also the case that if inhibition is the mechanism that underlies suppression effects, such effects should last as long as the inhibitory effects themselves (at least twenty minutes; e.g., Anderson, Bjork, \& Bjork, 1994).

In a study of retrieval-induced forgetting by MacLeod and Macrae (2001), however, the memory effects of inhibitory processes diminished after a delay of 24 hours. Generally, the procedure of a retrieval-induced forgetting study consists of study, retrieval practice, and testing phases. After study of several category-exemplar pairs (e.g., fruitlemon, fruit-banana, and profession-accountant), the participants are asked to retrieve about half of the category members via guided retrieval practice (e.g., fruit-le___), with such practice pairs denoted as $\mathrm{Rp}+$ items. Members of the practiced categories that do not receive retrieval practice (e.g., banana) are called Rp- items, and members of nonpracticed categories are called Nrp items (e.g., account). Participants then are asked to recall members of each category. Not surprisingly, $\mathrm{Rp}+$ items are better recalled than both $\mathrm{Rp}-$ and Nrp items. The surprising result is that Rp-items are more poorly recalled than are baseline Nrp items. Such a finding is referred to as retrieval-induced forgetting. In Experiment 1 of MacLeod and Macrae's study, participants were tested either immediately or one day after retrieval practice. Retrieval induced forgetting was observed on the immediate test but not on the 24-hour delayed test. Conversely, Storm, Bjork, Bjork, and Nestojko (2007) found that inhibition effects can endure even after a delay of 24 hours. In the experimental design used by Storm et al., participants were asked to take both immediate and 24 hour delayed cued recall tests following the study and retrieval practice phases. Retrieval-induced forgetting was observed on both tests. Because of these contradictory findings, the issue of whether inhibition occurs after 24 hours in retrievalinduced forgetting paradigms remains unresolved.

Our previous study indicated that the effects of forgetting in the Think/No-Think paradigm can last for at least five minutes (Hotta \& Kawaguchi, 2006). Recall in the repeated-suppression condition was worse than in the baseline ( 0 repetition) condition, on both immediate and five-minute delayed cued recall tests. This result resembles the enduring forgetting seen in some retrieval-induced forgetting studies. However, intentional (stopping retrieval with effort) and unintentional forgetting (retrieval-induced forgetting) constitute different processes. It is therefore unclear whether intentional suppression has the same durability as unintentional forgetting. The main purpose of the current study was to examine whether the Think/No Think findings of Hotta \& Kawaguchi (2006) could be extended to a 24-hour delayed recall test.

The current study was also designed to examine the relationship between enduring forgetting and the forgetting strategy used during No-Think trials. Hertel and Calcaterra (2005) found that participants who spontaneously thought about something else in order to avoid thinking about specific unwanted memories could suppress those memories better 
than those who did not use this specific strategy. This finding suggests that thought substitution during the No-Think trials can influence the degree of forgetting observed. Following Hertel and Calcaterra, we examined the relationship between use of this strategy and degree of forgetting by comparing the performance of participants who used a self-initiated strategy of thinking about something else in order to suppress a target with the performance of those who used no clear strategy. It was expected that participants who used a clear strategy would show better suppression of the response words than those who did not.

\title{
EXPERIMENT
}

\begin{abstract}
METHOD
Participants and design

Twenty-four university students (19 females; age range =19-26) participated in this study. We manipulated instructions during the Think/No Think phase (response vs. suppression). Number of Cue Word Repetitions during the Think/No-Think phase $(0,4$, or 12$)$ and Test Timing (immediate or delayed) were manipulated within participants. In the baseline condition ( 0 repetitions), participants memorized the words during the memorization phase but did not see them during the Think/No-Think phase. The dependent variable here was the percentage of words correctly recalled in each condition.
\end{abstract}

\section{Materials}

Word pairs. Forty semantically unrelated noun pairs (e.g., physics-organ, head-plane) were assembled to serve as paired associates. This set included 10 filler and 30 critical pairs, consisting of six sets of pairs with five in each. The assignment of each set to each trial type was counterbalanced across participants.

Cue words. There were 30 cue words. Cue words on the immediate cued recall test were identical to those used on the delayed cued recall test but were randomly rearranged.

\section{Procedure}

The software package Presentation (version 9.70) controlled all experimental tasks. Each participant was tested individually.

Pair-Training phase. Participants first studied all of the word pairs. Each pair was presented for 6000 $\mathrm{ms}$, and participants studied the items until they could provide a right hand response when provided with the left hand stimulus as a cue.

After studying all 40 pairs, memory was assessed using cued recall tests. Each cue was presented on the screen until the participant responded or until $5200 \mathrm{~ms}$ had elapsed. If participants could not recall the correct response, it appeared as feedback in a blue font at the center of the screen (for $2000 \mathrm{~ms}$ ). Each of the 40 pairs was tested until participants could provide the correct response on two consecutive testing sessions.

\section{The Think/No-Think phase}

Participants received trials that presented the stimulus member of one of the pairs at the center of the screen, and required all participants to perform one of two tasks: For some stimuli, participants recalled and said aloud the corresponding response as quickly as possible (Respond trials); for others, they were asked to avoid thinking about the corresponding word (Suppress trials). Participants first practiced performing the TNT task on 22 filler trials. At the start of each trial, a small fixation cross appeared for $200 \mathrm{~ms}$. The cue word of a pair was then presented alone. On these filler trials, participants were generally asked to recall the response word for each cue as quickly as possible, and they had up to $3000 \mathrm{~ms}$ to do so ( 9 of the filler trials). However, 1 cue was identified as a "suppression" cue, for which they were instructed to avoid retrieving the associated item. Respond cues were presented twice each (18 trials) and the Suppression cue was presented 4 times. When participants responded incorrectly on a Respond trial, feedback was given. When they made 
Table 1. Items on the Strategy Questionnaire

Item 1 Tried not to think of the associated response, but then after the trial was over I made sure I still remembered the response word.

Item 2 Kept myself from saying the response word aloud, but kept repeating the response word myself to improve my memory for it.

Item 3 Kept myself from thinking about the original response word by thinking about something else (another word or image, for example).

Item $4 \quad$ Kept myself from thinking about the response word by keeping my mind completely blank.

Note. Item 1 and 2: the degree of compliance with suppression instruction

Item 3: thought substitution strategy

any response at all on a Suppression trial, they were again instructed to avoid thinking of the response word.

After training on the TNT task, participants received a list of 10 cue words to review, and were told that these were the cues for which they were to suppress the associated response in the upcoming phase (Anderson $\&$ Green, 2001). They familiarized themselves with these cues such that they would recognize them and know that they were supposed to suppress the associated memory. To verify that participants could identify these cues as suppression cues, they were asked to identify 10 of the 21 cue words, including the 11 nonsuppression cues.

Participants then began the main TNT phase. They were told that if they saw one of the critical cues they just reviewed, they should avoid thinking about the corresponding response word (Suppress condition); otherwise, they were asked to recall the associated response (Respond condition). The cue words for suppressing or responding with a given word were either repeated 4 or 12 times each, for a total of 160 trials. In addition, 82 trials displayed filler word cues in the Respond task to create an overall tendency to respond, yielding a total of 242 trials that were presented in a random order.

\section{Testing phase}

For the immediate test, all participants were asked to recall the corresponding word for each cue. At the start of each trial, a small cross was displayed on the screen for $200 \mathrm{~ms}$, followed by presentation of a cue word. 30 cues were presented individually until the participant responded, or up to a maximum duration of $4000 \mathrm{~ms}$ each. All participants were then asked to return to the laboratory after 24 hours, to take the same cued recall test albeit with the item order randomly rearranged.

Strategy question. After final cued recall test, we asked participants to fill out a questionnaire concerning suppression strategies. Table 1 lists the questionnaire items (Hertel \& Calcaterra, 2005). Each item was rated on the following scale: 0 (never), 1 (rarely), 2 (sometimes), 3 (frequently), and 4 (very frequently). Participants were instructed to circle the number that represented their use of the strategy described. As shown in the Note of Table 2, we classified the participants into the two groups based on their responses to questionnaire item 3: The participants who thought about something else in order to avoid thinking about the target words (with-strategy group; scores above 3 on item 3 ) and those who clearly did not use this strategy (without-strategy group; score of 0 on item 3 ).

\section{RESUlTS AND DISCUSSION}

\section{Recall of target words}

Participants were not aware that they would have to take a recall test until the time came to do so. All participants reportedly complied with the suppression instructions (scores of 0 or 1 on questionnaire items 1 and 2). 
Immediate test condition

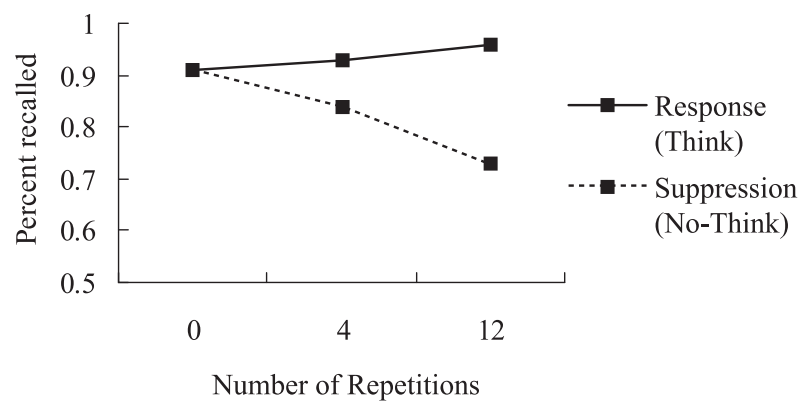

Delayed test condition

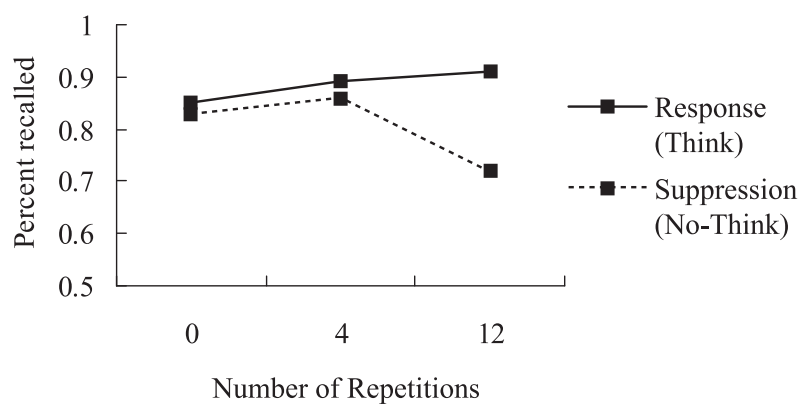

Fig. 1. Mean recall percentage as a function of Instruction, Number of Repetitions, and Testing Timing conditions. Figure above shows immediate test condition and figure below shows delayed test condition.

Figure 1 shows mean proportion of words recalled as a function of Instruction, Number of Repetitions and Test timing. Test timing conditions were analyzed separately because the recall results of both test types seem to be dependent for each.

Immediate recall test. A 2 (Instruction: response vs. suppression) $\times 3$ (Number of Repetitions: 0, 4, or 12) analysis of variance (ANOVA) revealed a main effect of Instruction, $F(1,23)=14.93, M S e=.71, p<.001$, with recall in the response condition $(M=.93, S D=.11)$ being significantly greater than recall in the suppression condition $(M=.83, S D=.18)$. The interaction between Instruction and Number of Presentations was also significant, $F(2,46)=6.72, M S e=.62, p<.01$. There was no significant effect of Number of Repetitions in the response condition, $F(2,92)=.63, M S e=.60, n s$, a finding that is inconsistent with previous research. However, there was a significant effect of Number of Repetitions in the suppression condition, $F(2,92)=8.66, M S e=.60$, $p<.001$. Recall in the 12-suppression condition $(M=.73, S D=.24)$ was worse than in the $0-(M=.91, S D=.14 ; t(92)=4.11, p<.0001)$ and $4-(M=.84, S D=.17 ; t(92)=2.62$, $p<.05)$ suppression conditions. 
Table 2. Mean Recall as a Function of Group, Instruction, Number of Repetitions, and Testing Timing Conditions

\begin{tabular}{ccccccc}
\hline & \multicolumn{3}{c}{ Immediate } & \multicolumn{3}{c}{ Delayed } \\
\cline { 2 - 3 } \cline { 6 - 7 } & 0 & 4 & 12 & 0 & 4 & 12 \\
\hline With-strategy & & & & & & \\
Response & $.92(.13)$ & $.94(.10)$ & $.99(.06)$ & $.86(.19)$ & $.89(.16)$ & $.92(.13)$ \\
Suppression & $.92(.13)$ & $.80(.20)$ & $.62(.22)$ & $.82(.17)$ & $.82(.21)$ & $.65(.15)$ \\
Without-strategy & & & & & & \\
Response & $.89(.16)$ & $.93(.10)$ & $.94(.14)$ & $.84(.18)$ & $.89(.16)$ & $.89(.16)$ \\
Suppression & $.89(.16)$ & $.89(.10)$ & $.86(.20)$ & $.84(.15)$ & $.91(.10)$ & $.80(.16)$ \\
\hline
\end{tabular}

Note. 13 participants reported: "I thought about something else in order to avoid thinking of the response word (With-strategy: with thought substitution strategy). 11 participants reported: "I reasoned myself out of thinking about the response word." Many of them tried to keep a neutral mind. (Without-strategy: without thought substitution strategy). The standard error is shown inside parentheses.

\section{Delayed recall test}

An analysis of variance (ANOVA) confirmed that there was a main effect of Instruction on the delayed test, $F(1,23)=10.46, M S e=.60, p<.01$, with recall in the response condition $(M=.88, S D=.16)$ being significantly greater than recall in the suppression condition $(M=.80, S D=.17)$. The Instruction $\times$ Number of Presentations interaction was also significant, $F(2,46)=3.51, M S e=.75, p<.05$. As was the case for the immediate test, there was no significant effect of Number of Repetitions in the response condition, $F(2,92)=.86, M S e=.63$. For the suppression condition, however, there was a significant effect of Number of Repetitions, $F(2,92)=5.25, M S e=.63$, $p<.01$. Recall in the 12-suppression condition $(M=.72, S D=.17)$ was worse than in the $0-(M=.83, S D=.16 ; t(92)=2.37, p<.05)$ and $4-(M=.86, S D=.17 ; t(92)=2.37$, $p<.05)$ suppression conditions.

To summarize, recall in the 12-suppression condition was worse than in the baseline condition, whereas recall in the 12-response condition was better than baseline. We therefore replicated the suppression effect demonstrated by Anderson and Green (2001). However, the magnitude of forgetting was relatively small in the 4-suppressed cue presentation condition. This may be because more than 10 suppression repetitions are needed to successfully forget a specific memory (Depue, Banich, \& Curran, 2006).

\section{Effect of using strategies}

Our experiment showed that the inhibition effects observed in the Think/No-Think paradigm can lead to relatively durable forgetting. Yet there remains the problem of how participants actually tried to suppress thoughts about the target words. Therefore, the relationship between enduring forgetting and the strategy used during the No-Think trials was examined. This analysis focused on the suppression condition because there were no 
notable differences between the two groups in the response condition.

Immediate recall performance in the Suppression condition. A mixed design ANOVA (2 (Strategy: high vs. low $\times 3$ (Number of Repetitions: 0,4 , or 12)) confirmed that there was a main effect of Strategy, $F(1,22)=5.35, M S e=.82, p<.05$, with recall in the With-strategy group being significantly better than recall in the Without-strategy group. There was also a main effect of Number of Repetitions, $F(2,44)=5.95$, $M S e=.76, p<.01$, indicating that overall recall in the 12-suppression condition was less than in the $0-(t(23)=3.42, p<.01)$ and $4-(t(23)=2.19, p<.05)$ suppression conditions.

The Strategy $\mathrm{X}$ Number of Repetitions interaction was also significant, $F(2$, $44)=3.61, M S e=.76, p<.05$. There was a significant effect of Number of Repetitions in the With-strategy group, $F(2,44)=9.39, M S e=.76, p<.001$ : Recall in the 12suppression condition was worse than in the $0-(t(44)=4.50, p<.0001)$ and 4$(t(44)=2.70, p<.01)$ suppression conditions. On the other hand, there was no significant effect of Number of Repetitions for the Without strategy group, $F(2,44)=.17, M S e=.76$, $n s$.

Delayed recall performance in the Suppression condition. There was a main effect of Strategy $(F(1,22)=7.11, M S e=.50, p<.05)$, with recall in the With-strategy group being significantly better than that in the Without-strategy group. There was also a main effect of Number of Repetitions, $F(2,44)=4.35, M S e=.71, p<.05$. Recall in the 12suppression condition was less than in the $0-(t(23)=2.11, p<.05)$ and $4-(t(23)=2.85$, $p<.01)$ suppression conditions.

Although the interaction of Strategy $\times$ Number of Repetitions was not significant, $F(2,44)=.92, M S e=.71, n s$, the following analysis was conducted in order to examine the relationship between strategy use and amount of forgetting. There was a significant effect of Number of Repetitions only in the With-strategy group, $F(2,44)=3.98$, $M S e=.71, p<.05$ (Without-strategy group; $F(2,44)=1.29, M S e=.71, n s$ ). Recall in the 12 -suppression condition was less than in the $0-(t(44)=2.55, p<.01)$ and 4 $(t(44)=2.55, p<.01)$ suppression conditions.

The above findings confirmed our expectation that the With-strategy group would more successfully suppress targets compared to the Without-strategy group, although the magnitude of forgetting remained small in the 4-suppressed presentation condition compared to baseline. The current experiment not only suggests that the memory impairment observed in the Think/No-Think paradigm can last at least 24 hours, but also that forgetting increases when participants use the strategy of thinking about something else so as not to think about a target, especially when the target is suppressed at least 12 times.

\section{GENERAL DisCUSSION}

In this study, we found that the intentional suppression effect can endure for up to 24 hours. This effect could relate to use of a thought substitution strategy to avoid thinking about the target words. This idea supports the findings of Hertel and Calcaterra (2005), 
who reported that use of self-initiated thought substitution aids successful forgetting.

The observation that the inhibition effect can be relatively enduring contradicts the findings of MacLeod and Macrae (2001). There are at least two possible reasons for this discrepancy. First, the durable effect may be specific to intentionally and repeatedly stopping retrieval. That is, retrieval-induced forgetting reflects unintentional forgetting, whereas forgetting by deliberately stopping retrieval reflects intentional forgetting. In a retrieval-induced forgetting procedure, repeated retrieval of practiced exemplars from practiced categories $(\mathrm{Rp}+)$ is enhanced relative to exemplars from unpracticed categories (Nrp), whereas recall of unpracticed exemplars from practiced categories (Rp-) is impaired relative to Nrp. Successful recall of Rp+ may lead to suppression of $\mathrm{Rp}-$ without conscious awareness, irrespective of the degree of retrieval practice. Conversely, in the Think/No-Think procedure participants intentionally suppress items. This suppression becomes possible with considerable repetition of No-Think trials (considering that there was no difference between the 0 and 4 trial suppression conditions in our experiment). Intentionally and repeatedly stopping the retrieval of a specific memory (suppression training) may reflect the durability of the suppression effect. Overall, there may be key differences between intentional and unintentional inhibition processes that could bear upon the durability of forgetting.

Second, it is also the case that the experimental design of the present study differed from that of MacLeod and Macrae (2001). Their design did not include an immediate test. Storm et al. (2007) indicated that testing the same material again after an interval can lead to long-term retrieval-induced forgetting. Therefore, the 24 hour intentional suppression effect that we observed here could have been affected by the immediate test: The suppression items were recalled less well than the baseline items retrieved for the first time since initial learning, and after 24 hours, prior retrieval could have benefited the retrieval of baseline items compared to a no prior retrieval condition. It has been suggested that no prior retrieval of baseline items (Nrp) can prevent retrieval-induced forgetting (MacLeod \& Macrae, 2001). When the Think/No-Think and test phases are separated by 24 hours, the intentional suppression effect might not be observed after a 24hour delay, as MacLeod and Macrae (2001) indicated. We cannot clearly state whether the durability of the intentional suppression effect observed in the current study is due to the inherent robustness of the effect or to repeated testing. These possibilities could be tested using a between-participants manipulation of retention interval, following MacLeod and Macrae (2001).

This study also examined the relationship between strategy use and durability of forgetting. As predicted, participants who explicitly thought about something else in order to suppress a specific memory succeeded in forgetting. It is of course necessary to further explore the efficacy of different strategies for forgetting (Hotta, Kawaguchi, \& Anderson, in preparation). It is significant that not only direct suppression of a specific memory with effort but also thought substitution can lead to stable and long-term forgetting. Addressing these implications is an interesting and important avenue for further research. 


\section{REFERENCES}

Anderson, M. C. 2003. Rethinking interference theory: Executive control and the mechanisms of forgetting. Journal of Memory \& Language, 49, 415-445.

Anderson, M. C., Bjork, E. L., \& Bjork, R. A. 1994. Remembering can cause forgetting: Retrieval dynamics in long-term memory. Journal of Experimental Psychology: Learning, Memory, and Cognition, 20, 1063-1087.

Anderson, M. C., \& Green, C. 2001. Suppressing unwanted memories by executive control. Nature, 410, 131-134.

Bjork, R. A. 1989. Retrieval inhibition as an adaptive mechanism in human memory. In H. L. Roediger III, \& F. I. M. Craik (Eds.) Variety of memory and consciousness: Essays in honor of Endel Tulving (pp. 309-330). Hillsdale, NJ: Erlbaum.

Depue, B. E., \& Banich, M. T., \& Curran, T. 2006. Suppression of Emotional and Nonemotional Content in Memory. Effects of Repetition on Cognitive Control. Psychological Science, 17, 441-447.

Hertel, P. T., \& Calcaterra, G. 2005. Intentional forgetting benefits from thought substitution. Psychonomic Bulletin \& Review, 12, 484-489.

Hotta, C., \& Kawaguchi, J. 2006. The effect of test delay on memory for suppressed item in Think/No-Think paradigm. The international symposium on "Inhibitory Processes in the Mind", in Kyoto.

Hotta, C., Kawaguchi, J., \& Anderson, M.C. (in preparation). Intentional suppression, not distraction, leads to long-term forgetting.

MacLeod, M. D., \& Macrae, C. N. 2001. Gone but not forgotten: The transient nature of retrieval-induced forgetting, Psychological Science, 12, 148-152.

Storm, B. C., Bjork, E. L., Bjork, R. A., \& Nestojko, J. F. 2006. Is retrieval success a necessary condition for retrieval-induced forgetting? Psychonomic Bulletin \& Review, 13, 1023-1027.

(Manuscript received November 30, 2007; Revision accepted January 5, 2009) 